\title{
The Pearl Oyster: from national icon to guardian of Qatar's marine environment
}

\author{
Alexandra Leitão-Ben Hamadou ${ }^{1}$, Zenaba Khatir ${ }^{1}$, Noora Al-Shamary¹, Hassan Hassan ${ }^{1}$, Zainab Hizam¹, Aisha Al- Ashwal ${ }^{1}$, Mark Chatting ${ }^{1}$, Shafeeq Hamza ${ }^{1}$ \\ Bruno Giraldes ${ }^{1}$, Diana Minardi², Tim Bean², Stéphane Bayen ${ }^{3}$ and Brett Lyons ${ }^{2}$
}

1:ESC-Qatar University, 2: Cefas, UK, 3: MCGill University, Canada

\section{Abstract}

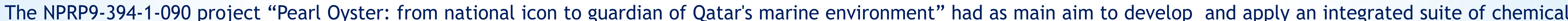

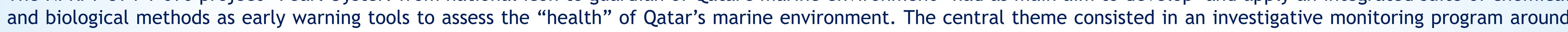

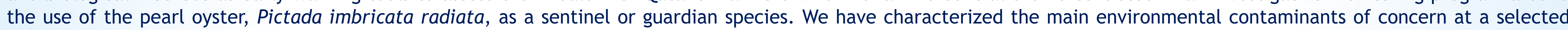

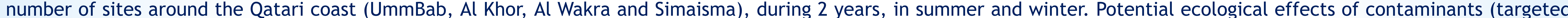

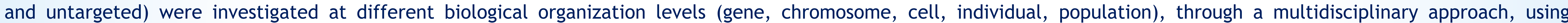

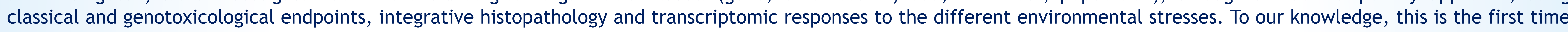

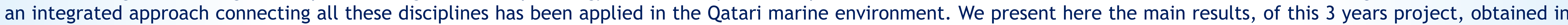

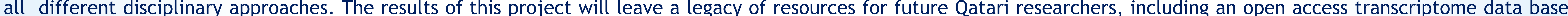

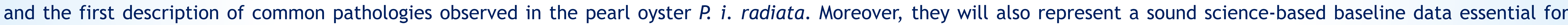

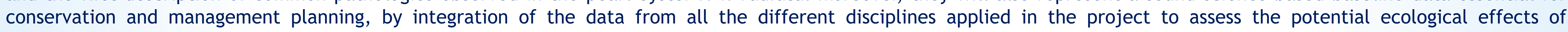
contaminants at different biological levels.

Key words: Marine Environmental Health; Marine Eco-genotoxicology; Integrative histopathology; Transcriptomics; Pearl oyster

\section{Chemical characterization}

Objectives: define the levels of trace metals and organic pollutants within the oyster $P_{.} \quad i$. radiata, water and sediment. Identify levels of emerging contaminants and sewage markers, with an emphasis on genotoxic compounds.

Main results:

$\checkmark$ A comprehensive set of data on the levels of metals, TPH, PAHs and CECs in the coastal environment of Qatar

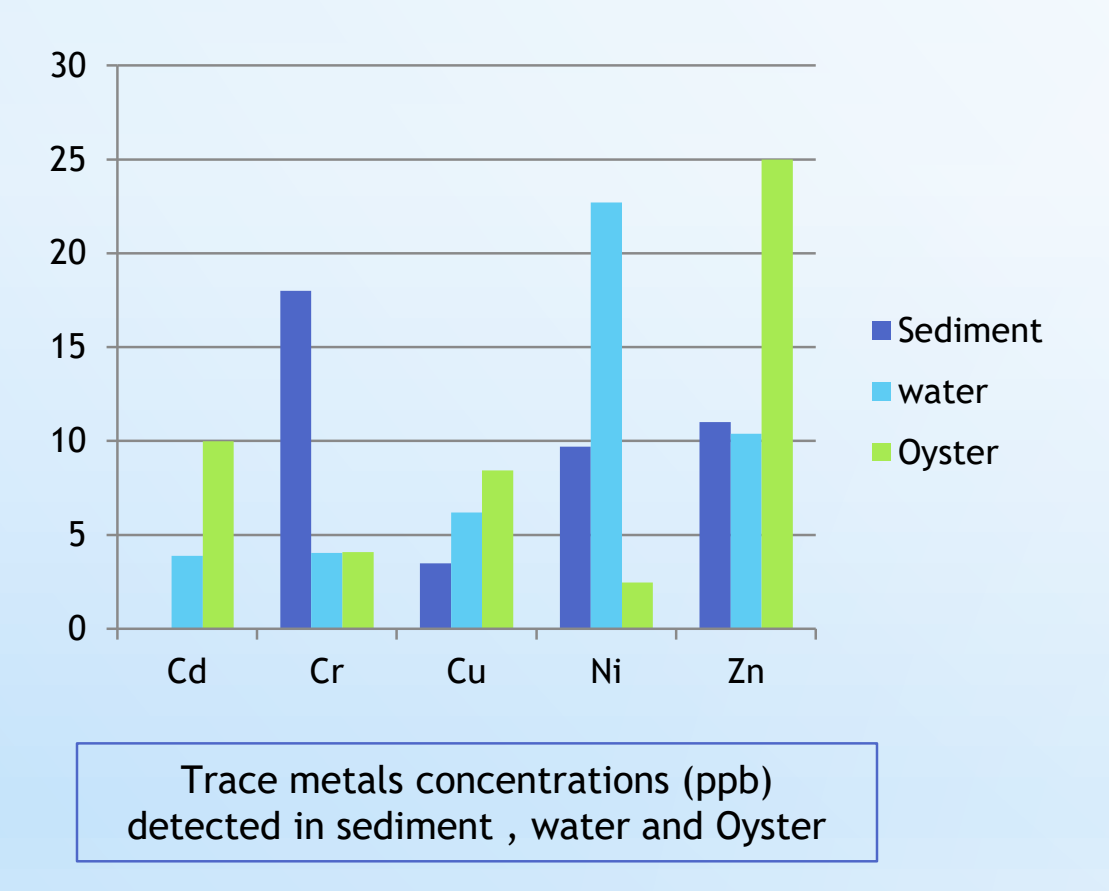

$\checkmark$ Novel methods for the combined targeted/non-targeted analysis of CECs in marine samples

The detection of new contaminants in marine samples

Highlighting the importance of complementing targeted analyses with a non-targeted approach

Objectives: to establish the genotoxicological profile of the populations of $P$. $i$. radiata

Main results:

$\checkmark$ The highest aneuploidy levels were observed in Simaisma, followed by Umm Bab
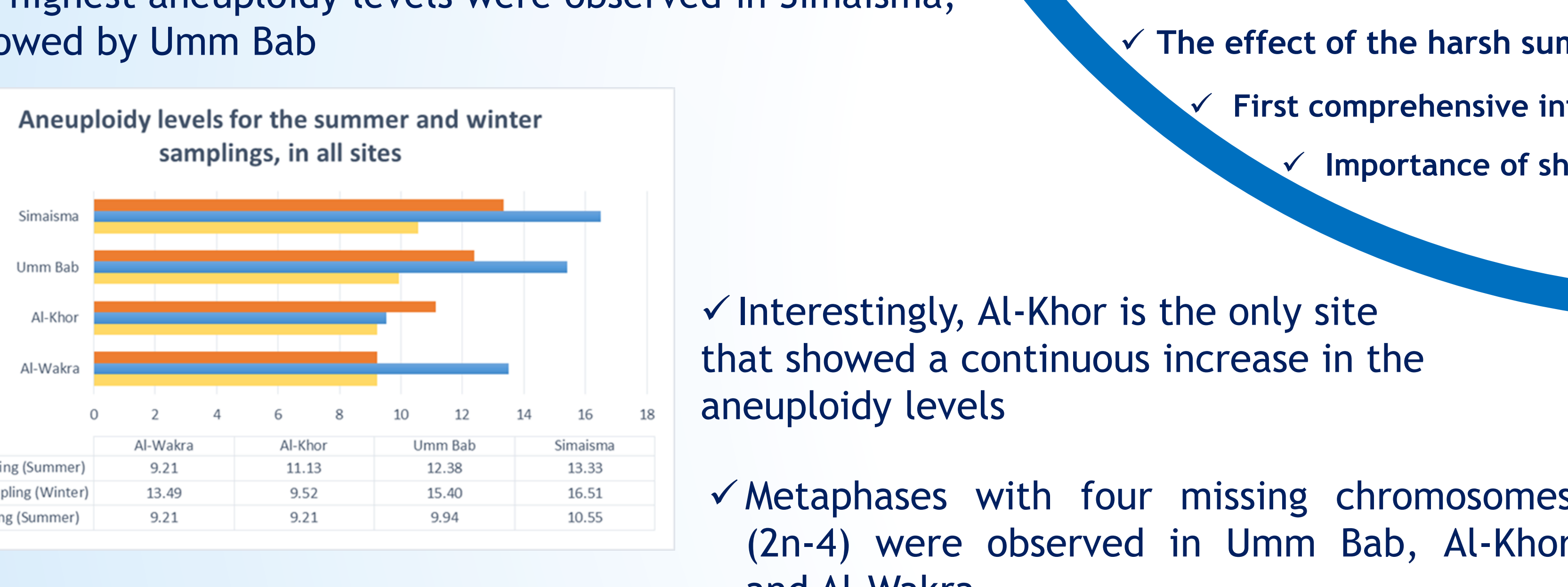
$(2 n-4)$ were observed in Umm Bab, Al-Kho and Al-Wakra.

$\checkmark$ Consistency of uncommon chromosomal gain -hyperdiploidy- pattern in all sampling rounds, up to $92 \%$ in some cases.

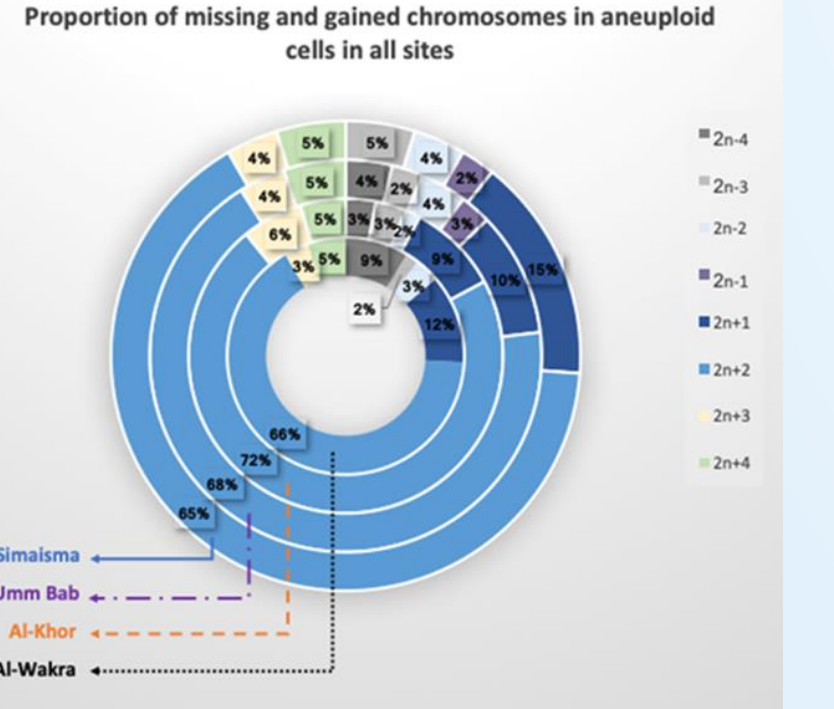

The identification of the extra and missing chromosomes (by performing the karyotypes of selected aneuploid cells after restriction enzyme banding) has showed a preferential lost in mainly 2 chromosomal pairs

\section{Genotoxicological characterization}

\section{Integrative histopathology}

Objectives: to investigate infectious and non-infectious disease conditions using histopathology Share key findings using digital pathology technology.

Main results:

$\checkmark$ A few oysters displayed an infection with a bucephalid trematode infection with multiple trematodes within the connective tissues surrounding the digestive gland in these individuals $(A, B, C, D)$.

$\checkmark$ A second trematode infection was also observed but at a lower prevalence within the tissues, 1 or 2 pathogens present in each section (A, B, C, D).

$\checkmark$ Metacercarial cysts of a an unknown metazoan were observed within the byssal gland in oysters sampled from Al Khor and Al Wakra (F).

\section{Ecological characterization}
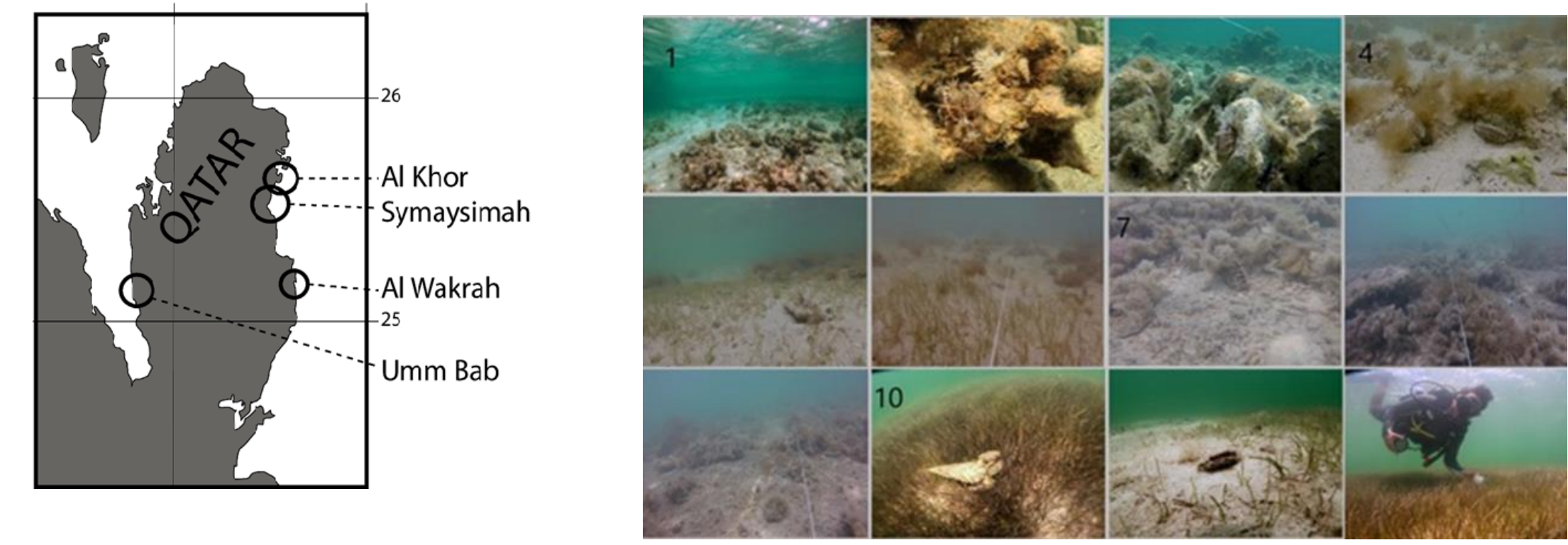

Main results:

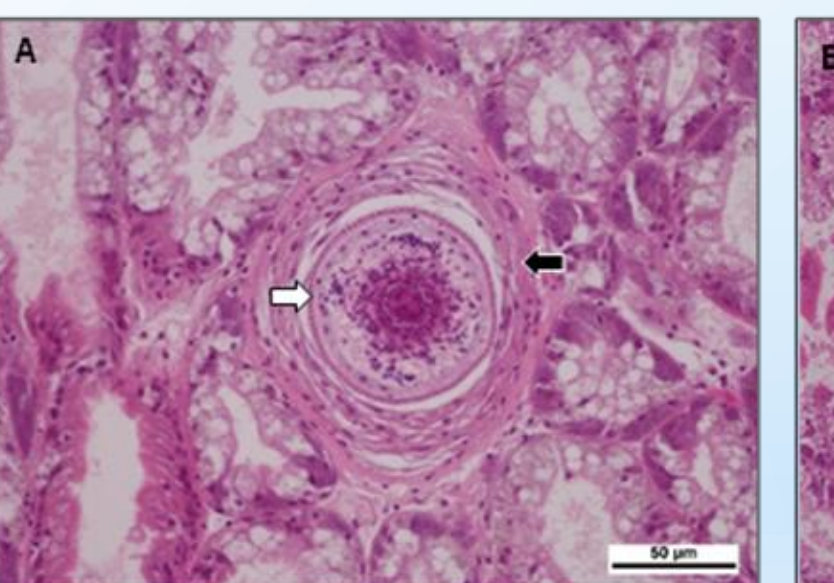

